\title{
Delaying surgery after neoadjuvant chemoradiotherapy in rectal cancer has no influence in surgical approach or short- term clinical outcomes
}

Nuno Figueiredo ${ }^{1 *}$, Sofoklis Panteleimonitis ${ }^{2,3^{*}}$, Sotiris Popeskou ${ }^{2}$, Jose F. Cunha ${ }^{1}$, Tahseen Qureshi ${ }^{2,4}$, Geerard L. Beets ${ }^{1,5}$, Richard J. Heald ${ }^{1}$, Amjad Parvaiz ${ }^{1,2,3}$

Affiliations:

1. Colorectal Surgery, Champalimaud Foundation, Lisbon, Portugal

2. Colorectal Surgery, Poole Hospital NHS Trust, Poole, United Kingdom

3. University of Portsmouth, School of Health Sciences and Social Work, Portsmouth, United Kingdom

4. Bournemouth University, Bournemouth, United Kingdom

5. Surgical Oncology, The Netherlands Cancer Institute, Amsterdam, The Netherlands

* These authors contributed equally to this paper

nunolfigueiredo@hotmail.com

sofoklis p@hotmail.com

salvator10@yahoo.com

jfilcunha@gmail.com

tas q@hotmail.com

g.beets@nki.nl

bill.heald@me.com

apcheema@yahoo.com

The Authors declare that they don't have any conflicts of interest. 


\section{Abstract}

Aims:

In rectal cancer, increasing the interval between the end of neoadjuvant chemoradiotherapy (CRT) and surgery could improve the pathological complete response (pCR) rates, allow fulldose neoadjuvant chemotherapy, and select patients with a clinical complete response (cCR) for inclusion in a "watch \& wait" program $(W \& W)$. However, controversy arises from waiting more than 8 to 12 weeks after CRT, as it might increase fibrosis around the total mesorectal excision (TME) plane potentially leading to technical difficulties and higher surgical morbidity. This study evaluates the type of surgical approach and short term post-operative outcomes in patients with rectal cancer that were operated before and after 12 weeks post CRT.

\section{Methods:}

Patients from three centres (two in the UK, one in Portugal) who received rectal cancer surgery following neoadjuvant CRT between 2007 and 2016 were identified from prospectively maintained databases. Preoperative CRT was given to patients with high risk for local recurrence (threatened CRM $\leq 2 \mathrm{~mm}$ or T4 in staging MRI). The baseline characteristics and surgical outcomes of patients that were operated $<12$ weeks and $\geq 12$ weeks after finishing CRT were analysed.

\section{Results:}

A total of 470 patients received rectal cancer surgery, of those 124 (26\%) received neoadjuvant CRT. Seventy-six patients (61\%) were operated $\geq 12$ weeks after end of neoadjuvant-CRT and $48<12$ weeks. Patients in the $\geq 12$ weeks cohort had a higher BMI (27 vs $25, p=0.030$ ) and lower lymph node yield (11 vs $14, p=0.001$ ). The remaining of the baseline characteristics were similar between the two groups (age, operating surgeon, gender, ASA grade, T stage, surgical approach, operation). Operation time, blood loss, conversion rate, length of stay, 30-day readmission rate, 30-day reoperation rate, anastomotic leak rate, 30-day mortality, CRM clearance, and ypT0 rates were similar between the two groups. Univariate and multivariate analysis showed that delaying surgery $\geq 12$ weeks did not affect morbidity and mortality.

\section{Conclusion:}

In our cohort, there was no difference in short term surgical outcomes between patients operated before or after 12 weeks following CRT. The type of surgical procedures and the proposed approach did not differ due to waiting after CRT. Delaying surgery by $\geq 12$ weeks is safe, feasible and does not result in higher surgical morbidity. 


\section{Introduction}

Neoadjuvant chemoradiotherapy (CRT) in rectal cancer can result in significant tumour regression and it has been advocated that it reduces the risk of local recurrence [1 - 4], even after correctly performed total mesorectal excision (TME) as described by RJ Heald in 1982 [5]. This has resulted in the widespread use of CRT for rectal cancer and whereas some units apply it more sparingly for cancers that threaten the mesorectal fascia (MRF) or invade adjacent structures [6], other units routinely use neoadjuvant CRT for all rectal cancers over cT2 or cN+ [7]. Increasing the interval between the end of neoadjuvant chemoradiotherapy (CRT) and surgery could improve the pathological complete response (pCR) rates, allow fulldose neoadjuvant chemotherapy, and select patients with a clinical complete response (CCR) for inclusion in a "watch \& wait" program (W\&W)

A significant proportion of patients receiving CRT will achieve pathological complete response $(\mathrm{pCR})$, heralding an excellent prognosis due to low local and distant recurrence rates [8, 9] . Several studies have shown that the rate of patients achieving PCR could be improved by increasing the interval between end of CRT and surgery [10 - 13] and this was also demonstrated in a meta-analysis [14]. This rationale is based on the principle that while DNA damage occurs during irradiation, cellular lysis occurs within the next weeks $[13,15,16]$.

Identifying clinical complete responders (CCR) that would be suitable for a W\&W strategy is also a matter of rising interest [17-19], adding to the rationale of waiting longer [20]. These CCR patients might benefit from non-operative management and intensive surveillance, avoiding permanent stomas, low anterior resection syndrome morbidity and adverse outcomes when quality of life issues are weighed [21].

Finally, there is a growing body of evidence that some locally advanced rectal cancer (LARC) patients might benefit from full neoadjuvant chemotherapy. With this in mind, the canonical 68 weeks waiting period after RT might not be enough to administer a full dose of induction or consolidation chemotherapy regimens [22 - 24].

However, there is no consensus on the optimal timing to operate [25]. Surgeons have been historically reluctant to delay surgery beyond 8 weeks as it might increase fibrosis around the TME plane, arguing that it could potentially lead to intra-operative technical difficulties and higher surgical morbidity.

While studies report that increasing the time interval to 8 weeks increased pCR rate without affecting patient morbidity [11, 26, 27], the GRECCAR-6 trial [28] showed that waiting until 11 weeks after CRT did not increase PCR after surgical resection and was associated with higher 
morbidity and worse specimen quality. Nevertheless, a recent population based study did not confirm the French observations [29].

The aim of this study was to compare short-term outcomes of rectal cancer surgery after an interval of less than 12 weeks after neoadjuvant CRT versus a longer interval. 


\section{Methods:}

Consecutive patients from three centres (two in the UK, one in Portugal) who underwent elective cancer resection surgery following neoadjuvant CRT between 2007 and 2016 were identified from prospectively maintained databases. Rectal cancer was defined as cancer present within $15 \mathrm{~cm}$ from the anal verge and confirmed in staging MRI.

Data collection began when the surgeons participating in this study started working in their respective units. This was from 2007 and 2012 for the UK centres and 2013 for the Portuguese centre. Informed consent was obtained from all patients.

\section{Peri-operative care}

Formal oncological and physical assessment was taken by all patients prior to surgery. Preoperative staging was performed by colonoscopy or CT colonography, computed tomography (CT) of the chest, abdomen, pelvis and magnetic resonance imaging (MRI) of the pelvis.

All patient findings were discussed in the multidisciplinary team meeting before treatment commencement. Preoperative CRT was given to patients with high risk for local recurrence (threatened CRM $\leq 2 \mathrm{~mm}$ or $\mathrm{T} 4$ in staging MRI).

Patients included in the study had surgery performed by three colorectal surgeons (AP is a UK Lapco ${ }^{\mathrm{TM}}$ trainer \& robotic proctor, having taught TQ and NF): one surgeon in each centre in the UK and one surgeon in Portugal. Surgery was open, laparoscopic, or robotic. Applied surgical modality was based on surgeon discretion. Minimally invasive TME was performed in a standardized fashion as described previously $[30,31]$. After pre-operative irradiation, all rectal resections with an anastomosis performed below the peritoneal reflection had a diverging ileostomy (or transverse colostomy) fashioned.

Post-operative care was standardised, with patients entering a routine enhanced recovery programme based on the one described by Kehlet and Wilmore [32]. Patients were discharged home per set criteria for discharge.

\section{Outcome assessment}

The baseline characteristics and surgical outcomes of patients that were operated $<12$ weeks and $\geq 12$ weeks after finishing CRT were analysed. Data was collected prospectively. Baseline characteristics analysed were sex, age, BMI, ASA grade, operating centre and T stage. Peri-operative data collected included operative time, estimated blood loss, surgical approach (open vs laparoscopic vs robotic), operation performed and conversion to open for 
laparoscopic and robotic procedures (defined as any incision needed to either mobilise the colon or rectum or ligate the vessels). Post-operative clinical and pathological data examined included length of stay, 30-day readmission, 30-day reoperation, 30-day mortality, anastomotic leak, lymph node yield, ypT0 rate and circumferential resection margin (CRM) clearance.

\section{Patient selection}

No specific pre-CRT selection criteria were used to decide whether patients were operated $<12$ or $\geq 12$ weeks after finishing CRT. Decision on time to operate following CRT completion was based on a shared-decision making process, including patient's preferences and multidisciplinary team decision. One centre had an ongoing program of deferral of surgery and offered patients to enter a "watch and wait" approach in case of a cCR.

The 12 week cut-off point for group analysis was decided upon lack of surgical and shortterm outcomes data beyond this point, in published trials [11, 26-28]. Additionally, when considering a "Watch and Wait" surveillance program, the final decision of proposing this nonoperative management usually is taken at restaging around 12 weeks.

\section{Statistical analysis}

IBM SPSS version 22 (SPSS Inc., Chicago, IL, USA) and Microsoft Excel 2010 ${ }^{\mathrm{TM}}$ were used for the statistical analysis. Non-parametric data was expressed as median with interquartile range. Baseline demographic and clinical characteristics were compared using $x^{2}$ test or Fishers exact test for categorical variables and Mann-Whitney $U$ test for continuous variables. $P$ values of $<0.05$ were considered statistically significant. Univariate binary logistic regression analysis was performed to assess whether operative treatment delay ( $<12$ or $\geq 12$ weeks) affected morbidity and mortality, with morbidity and mortality defined as the presence of any of the following outcomes: 30-day reoperation, 30-day readmission, anastomotic leak and 30day mortality. Following this, a multivariate model was applied were operative treatment delay was adjusted for all clinically relevant variables. These included: operating centre, age, BMI, sex and ASA grade. Age, BMI and ASA grade were included as continuous variables for the purpose of logistic regression analysis, with operating centre, (multinomial) and sex (binary) representing categorical variables. The constant was included in the analysis model and analysis was performed on IBM SPSS version 22. Data in logistic regression analysis are presented as odds ratio, $95 \%$ confidence interval and $p$ value. 


\section{Results}

Four hundred and seventy patients underwent rectal resection surgery by the three surgeons included in this study in three participating units. Neoadjuvant CRT was given to 124 (26\%) of those patients, all of whom were included in the study. Seventy-six patients (61\%) were operated $\geq 12$ weeks after end of CRT and $48(39 \%)<12$ weeks, as shown in the histogram - Figure 1. Median interval between CRT and surgery was $7[6,11]$ weeks for the first group, and $15[12,100]$ weeks for the latter.

\section{Baseline characteristics}

The baseline characteristics of the two groups are summarised in table 1. With the exception of BMI, which was higher in the $\geq 12$ weeks cohort (27 [IQR 24-29] vs 25 [IQR 22-28], $p=0.030$ ), the baseline characteristics of the two groups were broadly comparable.

\section{Peri-operative characteristics and outcomes}

The peri-operative characteristics of the two groups are summarised in table 2. Operative time, estimated blood loss and conversion rate were not different. The majority of the operations were performed laparoscopically in both groups and there were no differences between the two groups in terms of surgical approach and procedure.

\section{Post-operative clinical and pathological outcomes}

The post-operative characteristics of the two groups are summarised in table 3 . There were no differences in any of the post-operative clinical outcomes (length of stay, 30-day readmission rate, 30day reoperation rate, anastomotic leak rate, 30 -day mortality rate) between the two cohorts. In terms of pathological outcomes, CRM clearance and ypTO rates were similar between the two groups but lymph node yield was less in the $\geq 12$ weeks group (11 [IQR 8-15] vs 14 [IQR 12-19], $p=0.001$ ). There was a total of five 30-day reoperations and two 30-day mortalities. Reoperations included in the less than 12-week group a patient that received a laparotomy for fascial dehiscence and a patient that had a revision of a prolapsed stoma. In the over 12 weeks group reoperations included a patient that had a laparoscopy for an abdominal abscess, a laparotomy for an anastomotic leak and a Hartman's for an anastomotic leak. Both 30-day mortalities were in the less than 12 weeks cohort and both were due to myocardial infractions. 
Logistic regression analysis for morbidity and mortality

Univariate logistic regression analysis showed that delaying surgery $\geq 12$ weeks did not affect morbidity and mortality. This was still the case in multivariate analysis when other clinically relevant factors were considered (operating centre, age, BMI, sex, ASA grade). Findings are summarized in table 4. 


\section{Discussion}

Optimal timing to surgery following neoadjuvant CRT is controversial [25]. Although most studies seem to agree on an advantage on delaying surgery, the exact timing of the delay is up for debate $[\mathbf{1 1}, \mathbf{1 3}, \mathbf{2 6}]$.

Increasing the interval between the end of neoadjuvant chemoradiotherapy (CRT) and surgery could be an interesting approach to improve the pathological complete response ( $p C R)$ rates [33], allow a group of patients to benefit from fulldose neoadjuvant chemotherapy [24, 34], and finally to select patients with a clinical complete response (cCR) for inclusion in a "watch \& wait" program [20, 21]. Due to this shift of treatment paradigm in rectal cancer, surgery is sometimes being delayed longer and longer, as represented in Figure 1. This long tail in the histogram is due to the time inherently needed to take decisions in a "Watch \& Wait" program: to operate a non-responder; or to operate a local "regrowth" after a clinical complete response.

In the present study we found no difference in the short term surgical outcomes, and morbidity and mortality, between patients operated before or after 12 weeks following CRT. The finding of a slightly higher BMl at the time of surgery ( 27 vs 25 ) in the $\geq 12$ weeks cohort could either be a chance finding, or could be related to patients having more time to recover and regain any weight loss after CRT.

Although the 3 surgeons performed a standardized TME, patients in the $\geq 12$ week group had a lower lymph node yield (14 vs $11, p=0.001$ ). Hypothetically this could be the delayed effect of CRT, in which increasing interval has an incremental effect on inducing fibrosis in lymphatic structures. However, this finding is not supported by any other studies [27, 28, 33].

It is also worth mentioning that the anastomotic leak rate appears to be higher in the over 12 weeks cohort ( 0 vs $5.7 \% p=0.547$ ). Even though we recognize the chance of a type two error due to the limited sample size of the study, the $p$ value indicates that there is an over $50 \%$ that this finding might be due to chance. 
The GRECCAR-6 trial [28] is the only randomized trial, comparing a 7 vs 11 week interval after CRT in 265 patients. The trial reports an increased postoperative morbidity for the longer interval $(44.5 \% \mathrm{vs}$ $32 \%, p=0.04$ ) and worse quality of mesorectal resection (complete mesorectum $78.7 \%$ vs $90 \%$, $\mathrm{p}=0.016$ ). Sphincter preservation, anastomotic leak and re-intervention rates were similar between the two groups. The reported increased morbidity was primarily due to medical complications, such as urinary complications, and were mostly relatively minor (Dindo II), not requiring re-intervention or a longer length of stay. In our study, delaying $\geq 12$ weeks did not affect morbidity and anastomotic leak rates, translating into a similar 30-day reoperation, length of hospital stay and 30-day readmission rate. A possible explanation in this result discrepancy regarding morbidity might be that although in the GRECCAR-6 trial overall morbidity was higher in the 11-week group, this was primarily due to medical complications and specifically urinary complications, which did not affect re-intervention rate or length of stay. In fact, looking at the Dindo classification, only Dindo II complications were higher in the 11-week group, with Dindo III, IV and V being higher in the 7-week group. Considering that our dataset focuses on Dindo IIIb or above complications, or complications resulting in a readmission, this might justify the difference in terms of reported morbidity. A 2016 meta-analysis of the available (non-randomized) series reported for the longer interval group no difference in RO resection rate, DFS or surgical complications, and an increase in pCR. [14]

Another study compared the short-term outcomes of an increasing number of extra cycles of chemotherapy (mFOLFOX6) after conventional neo-adjuvant CRT and before TME [33]. The study consisted of four groups, with the control group receiving surgery 6-8 weeks after CRT, and the three experimental groups having a median delay time of 11.1, 15.4 and 19.3 weeks after CRT respectively. The rate of $p C R$ increased with a longer delay ( $18 \%$ vs $25 \%$ vs $30 \%$ vs $38 \%, p=0.0036$ ), without affecting reported technical difficulty, blood loss, RO rate, anastomotic leak rate and overall post-operative complication rate. Although the report describes an increased rate of pelvic fibrosis on a subjective fibrosis scale, this did not lead to an increased perceived difficulty as judged by the surgeons, and was not associated with postoperative complications.

In the context of delaying surgery, it is worth mentioning the results of the Stockholm III trial [27], comparing the outcome of short course RT with immediate surgery, short course RT with delayed surgery, and traditional long course with delayed surgery. They reported a higher rate of general and surgical postoperative complications with the short course and immediate surgery as compared to the two other arms with delayed surgery. It should be noted however that the interval to surgery in this trial was only 4-8 weeks, much shorter than the intervals studied in the present report. 
The last decade has witnessed a rising interest in organ preservation, either through a W\&W policy for clinical complete responders after CRT [37], or through local excisions of the remaining scar [20]. Especially for the W\&W policy a complete clinical response might not be evident at 6-8 weeks, as the bowel wall can still be in the healing phase. In very good responders the interval can be extended to 8-12 weeks to improve the ability to diagnosis a clinical complete response [20]. Of the three centres in the present study one is actively offering the W\&W approach to clinical complete responders and is recruiting patients for the International Watch \& Wait Database (IWWD - www.iwwd.org). Some of the patients with good responses at 6-8 weeks will still have residual tumour at a second assessment and will require TME surgery after an interval of more than 12 weeks. One of the concerns of surgeons with this approach is that delayed TME surgery might become more difficult.

The results of our study document that even when surgery is performed beyond the canonical 6-8 weeks period, patients are not hindered by an increase in LOS, 30-day readmission, 30-day reoperation, or 30-day mortality. In terms of surrogate oncologic outcomes, we did not observe a difference in CRM clearance, despite a lower lymph node yield in the delayed surgery group.

The limitations of the present study are related to the retrospective design and the possible selection bias in the short and long delay groups. Although retrospective, the data was collected from prospectively maintained databases, minimizing observation bias, and all consecutive patients were included to minimize selection bias. Furthermore, compared to GRECCAR-6 trial [28] a smaller number of patients were included in our study, increasing the risk of type 2 error in any of the outcomes. Another limitation is that we have not reported minor post-operative complications (Clavien-Dindo III). This is because on our data sets we only record complications that result in reoperation (ClavienDindo grade Illb or more) or complications resulting in a readmission. As a result, minor complications (Clavien-Dindo grade I-II) or complications of resulting in reintervention (Clavien-Dindo grade IIla) are not recorded unless leading to a readmission. It is conceivable that the complications (Clavien-Dindo grade I-IIla) would be reflected in a prolonged hospital stay or higher readmission rate, which was not the case in this study. However, we do recognize that these complications may have significant consequences in the patient's quality of life, without necessary leading to a higher length of stay or readmission rate.

In summary, our study shows that delaying surgery after CRT by more than 12 weeks does not seem to compromise the short-term surgical outcome. In the context of organ preservation with a W\&W policy it seems therefore logical to extend the observation interval to delay surgery to $\geq 12$ weeks in patients with a good response to CRT. This allows a better assessment of the tumour biology and the healing of the bowel wall. More patients will become eligible for organ preservation and will retain a 
better quality of life by avoiding major surgery. Further research with larger sample sized studies are required to throw further light into whether delaying surgery after CRT affects the short-term surgical outcomes of rectal cancer patients. Furthermore, it is important for future studies to investigate the functional outcomes of patients having delayed vs non-delayed surgery following CRT. This is an area were there is very little evidence and could be an important deciding factor when consulting patients whether to delay or not delay their surgery.

\section{Acknowledgements:}

The Authors would like to acknowledge Dr Carlos Carvalho, Dr. Oriol Pares, and Dr. Ines Santiago for their clinical support. We would also like to thank Dr Hugo Domingos and Ms Karen Flashman for their support on data management.

Stengths: This study adds to the existing literature examining the safety of delaying surgery after CRT and is of special interest since it is a surgical cohort of patients operated according to a standardized TME technique that stretches the delay period to more than 12 weeks.

Limitations: Our study is not a randomized controlled trial, adding potential bias to the selection of patients in the two groups. We were not able to collect the reason for delay in all patients. 


\section{References:}

1. Swedish Rectal Cancer, T., et al., Improved survival with preoperative radiotherapy in resectable rectal cancer. N Engl J Med, 1997. 336(14): p. 980-7.

2. Kapiteijn, E., et al., Preoperative radiotherapy combined with total mesorectal excision for resectable rectal cancer. N Engl J Med, 2001. 345(9): p. 638-46.

3. Sebag-Montefiore, D., et al., Preoperative radiotherapy versus selective postoperative chemoradiotherapy in patients with rectal cancer (MRC CR07 and NCIC-CTG C016): a multicentre, randomised trial. Lancet, 2009. 373(9666): p. 811-20.

4. Sauer, R., et al., Preoperative versus postoperative chemoradiotherapy for locally advanced rectal cancer: results of the German CAO/ARO/AIO-94 randomized phase III trial after a median followup of 11 years. J Clin Oncol, 2012. 30(16): p. 1926-33.

5. Heald, R.J., E.M. Husband, and R.D. Ryall, The mesorectum in rectal cancer surgery--the clue to pelvic recurrence? Br J Surg, 1982. 69(10): p. 613-6.

6. Taylor, F.G., et al., Preoperative high-resolution magnetic resonance imaging can identify good prognosis stage I, II, and III rectal cancer best managed by surgery alone: a prospective, multicenter, European study. Ann Surg, 2011. 253(4): p. 711-9.

7. Benson, A.B., 3rd, et al., Rectal Cancer, Version 2.2015. J Natl Compr Canc Netw, 2015. 13(6): p. 719-28; quiz 728.

8. Maas, M., et al., Long-term outcome in patients with a pathological complete response after chemoradiation for rectal cancer: a pooled analysis of individual patient data. Lancet Oncol, 2010. 11(9): p. 835-44.

9. Park, I.J., et al., Neoadjuvant treatment response as an early response indicator for patients with rectal cancer. J Clin Oncol, 2012. 30(15): p. 1770-6.

10. Garcia-Aguilar, J., et al., Optimal timing of surgery after chemoradiation for advanced rectal cancer: preliminary results of a multicenter, nonrandomized phase II prospective trial. Ann Surg, 2011. 254(1): p. 97-102.

11. Moore, H.G., et al., Rate of pathologic complete response with increased interval between preoperative combined modality therapy and rectal cancer resection. Dis Colon Rectum, 2004. 47(3): p. 279-86.

12. Kalady, M.F., et al., Predictive factors of pathologic complete response after neoadjuvant chemoradiation for rectal cancer. Ann Surg, 2009. 250(4): p. 582-9.

13. Glehen, O., et al., Long-term results of the Lyons R90-01 randomized trial of preoperative radiotherapy with delayed surgery and its effect on sphincter-saving surgery in rectal cancer. Br J Surg, 2003. 90(8): p. 996-8.

14. Petrelli, F., et al., Increasing the Interval Between Neoadjuvant Chemoradiotherapy and Surgery in Rectal Cancer: A Meta-analysis of Published Studies. Ann Surg, 2016. 263(3): p. 458-64.

15. Francois, Y., et al., Influence of the interval between preoperative radiation therapy and surgery on downstaging and on the rate of sphincter-sparing surgery for rectal cancer: the Lyon R90-01 randomized trial. J Clin Oncol, 1999. 17(8): p. 2396.

16. Bujko, K., Timing of surgery following preoperative therapy in rectal cancer: there is no need for a prospective randomized trial. Dis Colon Rectum, 2012. 55(3): p. e31; author reply e31-2.

17. Habr-Gama, A., et al., Operative versus nonoperative treatment for stage 0 distal rectal cancer following chemoradiation therapy: Iong-term results. Ann Surg, 2004. 240(4): p. 711-7; discussion 7178.

18. Maas, M., et al., Wait-and-see policy for clinical complete responders after chemoradiation for rectal cancer. J Clin Oncol, 2011. 29(35): p. 4633-40.

19. Renehan, A.G., et al., Watch-and-wait approach versus surgical resection after chemoradiotherapy for patients with rectal cancer (the OnCoRe project): a propensity-score matched cohort analysis. Lancet Oncol, 2016. 17(2): p. 174-83.

20. Beets, G.L., N.F. Figueiredo, and R.G. Beets-Tan, Management of Rectal Cancer Without Radical Resection. Annu Rev Med, 2017. 68: p. 169-182.

21. Martens, M.H., et al., Long-term Outcome of an Organ Preservation Program After Neoadjuvant Treatment for Rectal Cancer. J Natl Cancer Inst, 2016. 108(12).

22. Garcia-Aguilar, J., et al., Organ preservation for clinical T2NO distal rectal cancer using neoadjuvant chemoradiotherapy and local excision (ACOSOG Z6041): results of an open-label, singlearm, multi-institutional, phase 2 trial. Lancet Oncol, 2015. 16(15): p. 1537-46.

23. Probst, C.P., et al., Extended Intervals after Neoadjuvant Therapy in Locally Advanced Rectal Cancer: The Key to Improved Tumor Response and Potential Organ Preservation. J Am Coll Surg, 2015. 221(2): p. 430-40. 
24. Nilsson, P.J., et al., Short-course radiotherapy followed by neo-adjuvant chemotherapy in locally advanced rectal cancer--the RAPIDO trial. BMC Cancer, 2013. 13: p. 279.

25. Glimelius, B., Optimal Time Intervals between Pre-Operative Radiotherapy or Chemoradiotherapy and Surgery in Rectal Cancer? Front Oncol, 2014. 4: p. 50.

26. Tulchinsky, H., et al., An interval $>7$ weeks between neoadjuvant therapy and surgery improves pathologic complete response and disease-free survival in patients with locally advanced rectal cancer. Ann Surg Oncol, 2008. 15(10): p. 2661-7.

27. Erlandsson, J., et al., Optimal fractionation of preoperative radiotherapy and timing to surgery for rectal cancer (Stockholm III): a multicentre, randomised, non-blinded, phase 3, non-inferiority trial. Lancet Oncol, 2017. 18(3): p. 336-346.

28. Lefevre, J.H., et al., Effect of Interval (7 or 11 weeks) Between Neoadjuvant Radiochemotherapy and Surgery on Complete Pathologic Response in Rectal Cancer: A Multicenter, Randomized, Controlled Trial (GRECCAR-6). J Clin Oncol, 2016.

29. Rombouts, A.J., et al., Treatment Interval between Neoadjuvant Chemoradiotherapy and Surgery in Rectal Cancer Patients: A Population-Based Study. Ann Surg Oncol, 2016. 23(11): p. 3593601.

30. Miskovic, D., et al., Standardization of laparoscopic total mesorectal excision for rectal cancer: a structured international expert consensus. Ann Surg, 2015. 261(4): p. 716-22.

31. Ahmed, J., et al., Standardized technique for single-docking robotic rectal surgery. Colorectal Dis, 2016. 18(10): p. O380-O384.

32. Kehlet, H. and D.W. Wilmore, Multimodal strategies to improve surgical outcome. Am J Surg, 2002. 183(6): p. 630-41.

33. Garcia-Aguilar, J., et al., Effect of adding mFOLFOX6 after neoadjuvant chemoradiation in locally advanced rectal cancer: a multicentre, phase 2 trial. Lancet Oncol, 2015. 16(8): p. 957-66.

34. Schrag, D., et al., Neoadjuvant chemotherapy without routine use of radiation therapy for patients with locally advanced rectal cancer: a pilot trial. J Clin Oncol, 2014. 32(6): p. 513-8.

35. Bustamante-Lopez, L., et al., Understanding the factors associated with reduction in the number of lymph nodes in rectal cancer patients treated by neoadjuvant treatment. Int J Colorectal Dis, 2017. 32(6): p. 925-927.

36. Gurawalia, J., et al., Less than 12 lymph nodes in the surgical specimen after neoadjuvant chemo-radiotherapy: an indicator of tumor regression in locally advanced rectal cancer? J Gastrointest Oncol, 2016. 7(6): p. 946-957.

37. Beets, G.L., et al., A new paradigm for rectal cancer: Organ preservation: Introducing the International Watch \& Wait Database (IWWD). Eur J Surg Oncol, 2015. 41(12): p. 1562-4. 